\title{
RIQUEZA DE ESPÉCIES DE FORMIGAS EDÁFICAS EM PLANTAÇÃO DE EUCALIPTO E EM MATA SECUNDÁRIA NATIVA
}

\author{
Sandra De Miranda Soares ${ }^{1}$ \\ Cidália Gabriela Santos Marinho ${ }^{1}$ \\ Terezinha Maria Castro Della Lucia ${ }^{1}$
}

\begin{abstract}
SPECIES RICHNESS OF SOIL ANT FAUNA IN AN EUCALYPT PLANTATION AND A SECOND GROWTH FOREST. This investigation was conducted to compare the soil ant fauna of an eucalypt plantation to that of a second growth forest; both areas located in the Zona da Mata of Minas Gerais, Brazil. Tuna fish, honey and cracker baits were used to collect ants along a transect and pitfall traps were distributed in central $900 \mathrm{~m}^{2}$ of each site. A total of 64 species belonging to 26 genera were collected in the 12 weekly samples; 54 species were captured in the forest and 45 in the Eucalyptus grandis plantation. A total of eight species presented frequencies above $10 \%$ in at least one area. Species richness was higher in the second growth forest. Additional studies should be considered for further generalizations of the results.

KEY WORDS. Brazilian atlantic forest, eucalypt, second growth forest, soil ant fauna
\end{abstract}

As formigas são abundantes na maioria dos ecossistemas terrestres, sendo freqüentemente consideradas ecologicamente dominantes (WILSON 1971; MAJER 1985). Elas apresentam uma diversidade de adaptações ecológicas e sociais que favorece sua ampla dispersão no ambiente, tais como grande variedade no padrão alimentar (Fowler et al. 1991) e nos habitats de nidificação (BENSON \& HARADA 1988). Diversos trabalhos destacam a importância das formigas como bio-indicadoras (MAJER 1985; ANDERSEN 1990; BURBIDGE et al. 1992; MAJER \& KOCK 1992; GADAGKAR et al. 1993). Esta importância está relacionada com vários fatores, dentre os quais, pode-se citar: (1) sua capacidade de interagir de várias maneiras com outras partes do ecossistema (MorAIs \& BENSON 1988; BURBIDGE et al. 1992), influenciando no padrão de distribuição e abundância de outros táxons (MAJER \& CAMER-PESCI 1991); (2) sua extrema abundância e diversidade; (3) sua importância funcional nas cadeias tróficas; (4) sua relativa facilidade de coleta; (5) sua alta sensibilidade às variáveis ambientais e (6) sua capacidade de reagir rapidamente a mudanças no ambiente (ANDERSEN 1990).

Os aspectos ecológicos dos formicídeos têm sido, portanto, utilizados em estudos comparativos de ecossistemas. Na maioria destes estudos, é proposto que a complexidade estrutural do habitat afeta a diversidade da formicifauna (CASTRO \& QueIroz 1987; BurbidGe et al. 1992; Oliveira \& Della luCia 1992; GADAGKAR et al. 1993).

1) Departamento de Biologia Animal, Universidade Federal de Viçosa. 36571-000 Viçosa, Minas Gerais, Brasil. 
Há evidências de que em áreas de plantação de eucalipto ocorre menor riqueza de espécies da formicifauna que em áreas de vegetação nativa (MAJER 1992). Verifica-se, no entanto, que, embora os eucaliptais já ocupem uma área de quatro milhões de hectares no Brasil (OHMART \& EDWARDS 1991), ainda há carência de trabalhos mais específicos sobre a sua ecologia, assim como dados comparativos com outras comunidades florísticas nativas, em diversas regiões do País.

O presente trabalho teve como objetivo fazer um estudo comparativo da formicifauna edáfica entre uma área com plantação de eucalipto e outra de mata secundária nativa.

\section{MATERIAL E MÉTODOS}

\section{Áreas de estudo}

Este trabalho foi conduzido no município de Viçosa, Minas Gerais, $20^{\circ} 45^{\prime} \mathrm{S}$, $42^{\circ} 50^{\prime} \mathrm{W}$, altitude $660 \mathrm{~m}$ (IBGE 1959), em uma área de plantação de eucalipto e outra de mata secundária nativa. A área de plantação de eucalipto consistia de cerca de 3,5 hectares, sendo constituída por uma única espécie, Eucalyptus grandis W. Hill ex Maiden, de quatro anos de idade. Não apresentava sub-bosque e o solo era coberto por espessa camada de serapilheira. $\mathrm{O}$ fragmento de mata secundária nativa, utilizado no presente estudo, situava-se próximo à área de plantação de eucalipto, distando $300 \mathrm{~m}$ desta. Abrangia, aproximadamente, 3 hectares, apresentava subbosque pouco denso e em seu interior existiam várias clareiras, onde podiam ser encontradas diversas espécies de plantas pioneiras.

\section{Técnicas de coleta}

$\mathrm{Na}$ coleta das formigas foram utilizadas armadilhas com iscas e armadilhas de solo modificadas de DOANE (1961). As armadilhas de iscas consistiram em tampas plásticas de $9 \mathrm{~cm}$ de diâmetro, onde eram colocadas, simultaneamente, pequenas porções de sardinha, mel e biscoito. Estas armadilhas eram distribuídas em intervalos de $10 \mathrm{~m}$, ao longo de um transecto de $90 \mathrm{~m}$, posicionado no centro das áreas de estudo, e deixadas no solo por um período de 90 minutos.

$\mathrm{Na}$ instalação das armadilhas de solo, foi delimitada uma grade de $900 \mathrm{~m}^{2}$ (30m X 30m) no centro de ambas as áreas de estudo. Esta grade foi dividida em nove quadrantes de $10 \mathrm{~m}^{2}$, sendo colocada uma armadilha no centro de cada um. A distância entre as armadilhas era, portanto, de $10 \mathrm{~m}$. Cada armadilha de solo era constituída de uma lata cilíndrica $(18,5 \mathrm{~cm}$ de altura $\times 8,3 \mathrm{~cm}$ de diâmetro) enterrada ao nível do solo. No interior desta lata era colocado um frasco coletor contendo solução de álcool $75 \%$. Sobre a superfície da lata, ao nível do solo, foi posicionado um funil de plástico direcionado para o centro do frasco coletor. O conjunto foi coberto por tampa de madeira sustentada por dois pinos e pintada de vermelho para proteger a armadilha e facilitar a localização da mesma nas áreas experimentais. A ativação desta armadilha ocorreu uma semana após sua instalação para diminuir as perturbações do solo causadas pela escavação, conforme o proposto por DELLA LUCIA et al. (1982) e BRUYN (1993). 
As coletas foram realizadas semanalmente, sempre no período diurno entre 8:00 e 12:00 horas, durante os meses de dezembro de 1995, janeiro e fevereiro de 1996, tendo sido obtido um total de 12 coletas. Em cada coleta, foi efetuada a troca do líquido coletor das armadilhas de solo.

Os espécimes coletados foram identificados a nível genérico e separados em morfoespécies. Nessa identificação, foram utilizadas chaves taxonômicas (HöLLDOBLER \& WILSON 1990). A identificação a nível específico foi efetuada, sempre que possível, pelo Dr. Jacques H.C. Delabie (Centro de Pesquisas do Cacau da CEPLAC, Itabuna, Bahia). Exemplares de todos os táxons coletados encontram-se depositados no Museu Regional de Entomologia da Universidade Federal de Viçosa.

\section{Análise dos dados}

Os dados obtidos foram utilizados para calcular as estimativas da riqueza de espécies através do método de Jackknife, conforme descrito por HeLTSHE \& FORRESTER (1983). Estas estimativas foram calculadas por meio de um programa de computador desenvolvido pelo professor Paulo de Marco Júnior (Departamento de Biologia Geral da Universidade Federal de Viçosa).

O método de Jackknife consiste na produção de pseudovalores com a retirada de cada uma das unidades amostrais tomadas (MANLY 1994; MAGURRAN 1995). A média destes pseudovalores é utilizada como estimativa para o parâmetro requirido (riqueza de espécies). Além disso, este método pode ser usado para associar limites de confiança à estimativa calculada e permite testes estatísticos utilizando a distribuição t de student (MANLY 1994). Neste trabalho, cada coleta semanal foi utilizada como uma unidade amostral nos cálculos das estimativas de Jackknife.

Para comparar a abundância das espécies nas duas áreas estudadas, foram utilizados os dados de freqüência de captura (número de armadilhas nas quais cada espécie foi coletada dividido pelo número total de armadilhas), como sugerido por BENSON \& HARADA (1988) e ANDERSEN (1990). Esse método evita a superestimativa das espécies com alta capacidade de recrutamento de operárias (LEAL \& LOPES 1992).

\section{RESULTADOS}

Um total de 64 espécies pertencentes a 26 gêneros e 6 subfamílias foi coletado neste estudo (Tab. I). A maioria das espécies obtidas pertence à subfamília Myrmicinae (31), sendo a subfamília Ponerinae representada por 16 espécies, Formicinae por 9, Dolichoderinae por 4, Ecitoninae por 3 e Pseudomyrmecinae por apenas uma espécie.

Nas armadilhas de solo, foi coletado um total de 55 espécies, distribuídas em 26 gêneros. Destas, 46 foram encontradas na mata, 38 na plantação de eucalipto e 29 foram coletas em ambas as áreas. Nas armadilhas de iscas, obteve-se 33 espécies de 16 gêneros, das quais 26 ocorreram na mata, 26 na plantação de eucalipto e 18 foram comuns às duas áreas (Tab. I). 
Tabela I. Freqüência de captura das espécies de Formicidae nas armadilhas de solo do tipo Doane e nas armadilhas de iscas, em mata secundária e plantação de eucalipto de Viçosa, Minas Gerais.

\begin{tabular}{|c|c|c|c|c|}
\hline \multirow[t]{2}{*}{ Frequêencias (\%) } & \multicolumn{2}{|c|}{ Doane } & \multicolumn{2}{|c|}{ Isca } \\
\hline & Mata & Eucaliptal & Mata & Eucaliptal \\
\hline \multicolumn{5}{|l|}{ Dolichoderinae } \\
\hline Dolichoderus imitator Emery, 1894 & 0,0 & 0,9 & 0,0 & 0,0 \\
\hline Linepithema humile (Mayr, 1866) & 0,0 & 0,0 & 0,8 & 0,8 \\
\hline Linepithema sp. & 0,9 & 0,0 & 0,0 & 0,0 \\
\hline Tapinoma melanocephalum (Fabricius, 1793) & 0,9 & 0,0 & 3,3 & 0,8 \\
\hline \multicolumn{5}{|l|}{ Ecitoninae } \\
\hline Eciton burchelli (Westwood, 1842) & 0,9 & 0,0 & 0,0 & 0,0 \\
\hline Labidus mars (Forel, 1912) & 0,9 & 0,0 & 0,0 & 0,0 \\
\hline Labidus praedator (Fr. Smith, 1858) & 4,6 & 2,8 & 0,0 & 0,0 \\
\hline \multicolumn{5}{|l|}{ Formicinae } \\
\hline Brachymyrmex sp. & 4,6 & 1,8 & 2,5 & 4,2 \\
\hline Camponotus cingulatus (Mayr, 1862) & 11,1 & 2,8 & 5,0 & 4,2 \\
\hline Camponotus crassus Mayr, 1862 & 0,9 & 2,8 & 6,7 & 2,5 \\
\hline Camponotus grupo agra (Fr. Smith, 1858) & 0,9 & 0,9 & 0,0 & 5,0 \\
\hline Camponotus rufipes (Fabricius, 1775 ) & 17,6 & 7,4 & 18,3 & 33,3 \\
\hline Camponotus sericeiventris (Guerin, 1838) & 0,0 & 0,0 & 1,7 & 0,0 \\
\hline Camponotus sp. 1 & 0,0 & 0,0 & 0,8 & 0,0 \\
\hline Camponotus sp. 2 & 0,0 & 0,0 & 1,7 & 0,0 \\
\hline Paratrechina sp. & 0,0 & 0,9 & 0,0 & 5,8 \\
\hline \multicolumn{5}{|l|}{ Myrmicinae } \\
\hline Acromyrmex subterraneus subterraneus Forel, 1893 & 20,4 & 1,8 & 5,8 & 0,8 \\
\hline Apterostigma sp. & 9,3 & 0,9 & 0,0 & 0,0 \\
\hline Atta sexdens rubropilosa Forel, 1908 & 19,4 & 5,5 & 4,2 & 0,0 \\
\hline Crematogaster sp. & 0,9 & 0,0 & 11,7 & 1,7 \\
\hline Cyphomyrmex salvini Forel, 1899 & 8,3 & 1,8 & 0,0 & 0,0 \\
\hline Cyphomyrmex olitor Forel, 1893 & 0,9 & 0,0 & 0,0 & 0,0 \\
\hline Megalomyrmex goeldii Forel, 1912 & 0,0 & 0,0 & 0,8 & 0,0 \\
\hline Megalomyrmex myops Santschi, 1925 & 0,9 & 0,9 & 0,0 & 0,0 \\
\hline Megalomyrmex silvestrii Wheeler, 1909 & 0,0 & 0,9 & 0,0 & 0,0 \\
\hline Megalomyrmex grupo pusillus & 0,0 & 0,9 & 0,0 & 0,0 \\
\hline Pheidole sp. 1 & 0,0 & 0,0 & 0,8 & 0,0 \\
\hline Pheidole sp. 2 & 7,4 & 4,6 & 3,3 & 1,7 \\
\hline Pheidole sp. 3 & 0,0 & 0,0 & 5,8 & 5,0 \\
\hline Pheidole sp. 4 & 0,0 & 0,9 & 0,0 & 0,0 \\
\hline Pheidole sp. 5 & 1,8 & 0,9 & 5,0 & 3,3 \\
\hline Pheidole sp. 6 & 6,5 & 0,0 & 6,7 & 2,5 \\
\hline Pheidole sp. 7 & 0,9 & 1,8 & 0,0 & 2,5 \\
\hline Pheidole sp. 8 & 2,8 & 14,8 & 2,5 & 3,3 \\
\hline Pheidole sp. 9 & 0,0 & 5,5 & 0,0 & 0,8 \\
\hline Pheidole sp. 10 & 0,0 & 0,9 & 0,0 & 0,0 \\
\hline Pheidole sp. 11 & 0,0 & 0,0 & 0,0 & 1,7 \\
\hline Pheidole sp. 12 & 0,0 & 0,0 & 1,7 & 4,2 \\
\hline Solenopsis sp. 1 & 0,9 & 0,9 & 0,0 & 0,0 \\
\hline Solenopsis sp. 2 & 17,6 & 12,0 & 3,3 & 18,3 \\
\hline Solenopsis sp. 3 & 4,2 & 0,9 & 0,8 & 1,7 \\
\hline Strumigenys sp. 1 & 0,0 & 0,9 & 0,0 & 0,0 \\
\hline Strumigenys sp. 2 & 1,8 & 0,0 & 0,0 & 0,0 \\
\hline Trachymyrmex sp. 1 & 26,8 & 25,0 & 2,5 & 1,7 \\
\hline Trachymyrmex sp. 2 & 0,9 & 0,0 & 0,0 & 0,0 \\
\hline Trachymyrmex sp. 3 & 0,0 & 2,8 & 0,0 & 0,0 \\
\hline Wasmannia auropunctata (Roger, 1863) & 39,8 & 13,0 & 21,7 & 1,7 \\
\hline \multicolumn{5}{|l|}{ Ponerinae } \\
\hline Anochetus targionii Emery, 1894 & 0,9 & 0,0 & 0,0 & 0,0 \\
\hline Ectatomma edentatum Roger, 1863 & 55,5 & 0,9 & 14,2 & 0,0 \\
\hline Ectatomma permagnum Forel, 1908 & 0,9 & 0,0 & 0,0 & 0,0 \\
\hline Gnamptogenys acuminata Emery, 1896 & 8,3 & 3,7 & 0,0 & 0,0 \\
\hline Gnamptogenys sp. & 0,9 & 1,8 & 0,0 & 0,0 \\
\hline
\end{tabular}


Tabela I. Continuação.

\begin{tabular}{|c|c|c|c|c|}
\hline \multirow[t]{2}{*}{ Freqüẽncias (\%) } & \multicolumn{2}{|c|}{ Doane } & \multicolumn{2}{|c|}{ Isca } \\
\hline & Mata & Eucaliptal & Mata & Eucaliptal \\
\hline Gnamptogenys striatula Mayr, 1883 & 4,6 & 10,2 & 0,0 & 0,0 \\
\hline Odontomachus chelifer (Latreille, 1802) & 1,8 & 7,4 & 0,0 & 3,3 \\
\hline Odontomachus minutus Emery, 1894 & 2,8 & 1,8 & 0,0 & 0,8 \\
\hline Hypoponera sp. 1 & 1,8 & 1,8 & 0,0 & 0,0 \\
\hline Hypoponera sp. 2 & 0,9 & 0,0 & 0,0 & 0,0 \\
\hline Hypoponera sp. 3 & 2,8 & 0,0 & 0,0 & 0,0 \\
\hline Hypoponera sp. 4 & 0,9 & 0,0 & 0,0 & 0,0 \\
\hline Pachycondyla obscuricornis (Emery, 1890) & 0,9 & 0,0 & 0,0 & 0,0 \\
\hline Pachycondyla harpax (Fabricius, 1804) & 7,4 & 6,5 & 0,0 & 0,0 \\
\hline Pachycondyla marginata (Roger, 1861) & 8,3 & 0,0 & 0,0 & 0,0 \\
\hline $\begin{array}{l}\text { Pachycondyla striata Fr. Smith, } 1858 \\
\text { Pseudomyrmecinae }\end{array}$ & 5,5 & 22,2 & 4,2 & 0,8 \\
\hline Pseudomyrmex tenuis (Fabricius, 1804) & 0,9 & 0,0 & 0,0 & 0,0 \\
\hline $\mathrm{N}^{\circ}$ de espécies & 46 & 38 & 26 & 26 \\
\hline $\mathrm{N}^{0}$ total de armadilhas & 108 & 108 & 120 & 120 \\
\hline
\end{tabular}

Formigas das subfamílias Ecitoninae e Pseudomyrmecinae foram coletadas apenas nas armadilhas de solo. Além disso, o número de espécies representantes da subfamília Ponerinae foi quatro vezes maior nas armadilhas de solo que nas de iscas. Por outro lado, as armadilhas de iscas coletaram nove espécies não capturadas nas armadilhas tipo Doane.

Os gêneros de formigas predominantes em número de espécies foram Pheidole, com 12 espécies, e Camponotus, com sete. A predominância de Pheidole foi mais pronunciada no eucaliptal (11 espécies) do que na mata (7 espécies). O contrário ocorreu com o gênero Camponotus, que foi representado por sete espécies na mata e quatro no eucaliptal.

As espécies que se destacaram em termos de freqüência de captura foram: Ectatomma edentatum e Wasmannia auropunctata na mata, ambas com mais de $30 \%$ de frequiência; e Camponotus rufipes e Solenopsis sp. 2 no eucaliptal, que apresentaram uma freqüência superior a 15\% (Tab. II). Algumas espécies e subespécies representantes da tribo Attini também foram obtidas em alta freqüência na mata. São elas: Trachymyrmex sp. 1, Acromyrmex subterraneus subterraneus e Atta sexdens rubropilosa (Tab. II).

Tabela II. Espécies de formigas coletadas nas áreas de mata e/ou plantação de eucalipto com frequeência superior a $10 \%$.

\begin{tabular}{lcc}
\hline \multicolumn{1}{c}{ Táxons } & \multicolumn{2}{c}{ Freqüéncia (\%) } \\
\cline { 2 - 3 } & Mata & Eucaliptal \\
\hline Acromyrmex subterraneus subterraneus & 12,7 & 1,3 \\
Alta sexdens rubropilosa & 11,4 & 2,6 \\
Camponotus rufipes & 18,0 & 21,0 \\
Ectatomma edentatum & 33,8 & 0,4 \\
Pachycondyla striata & 4,8 & 11,0 \\
Solenopsis sp. 2 & 10,1 & 15,3 \\
Trachymyrmex sp. 1 & 14,0 & 12,7 \\
Wasmannia auropunctata & 30,3 & 7,0 \\
\hline
\end{tabular}


O número de espécies (S), estimado pelo método de Jackknife para as áreas de mata e eucaliptal, nas armadilhas Doane e de iscas, é ilustrado na figura 1.

O número de espécies foi significativamente maior na mata que no eucaliptal nas armadilhas de solo. Tal diferença não foi verificada nas armadilhas de iscas (Fig. 1).

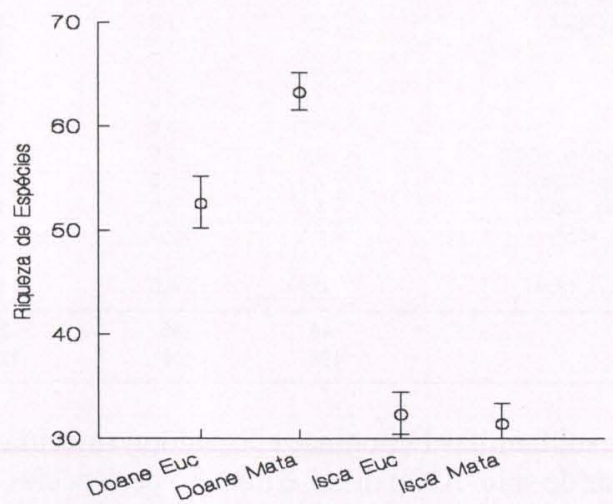

Fig. 1. Riqueza de espécies estimada pelo método de Jackknife para as áreas de mata e eucaliptal, nas armadilhas de solo (Doane) e de iscas (as barras representam o intervalo de confiança de $95 \%$ para as estimativas de Jackknife).

\section{DISCUSSÃO}

Myrmicinae foi a subfamília dominante em ambos ambientes tanto em número de gêneros quanto em número de espécies. Este resultado assemelha-se aos de trabalhos anteriormente realizados (OLIVEIRA \& DELLA LUCIA 1992; BONNET \& LOPES 1993; BELSHAW \& BOLTON 1994; ARMBRECHT 1995).

A raridade da subfamília Pseudomyrmecinae nas armadilhas pode estar relacionada com o fato de a maioria das espécies destes formicídeos apresentar hábitos arborícolas (JAFFÉ 1993). A predominância de Pheidole e Camponotus em número de morfoespécies, verificada neste trabalho, assemelha-se aos resultados de LOPES \& LEAL (1991) e ARMBRECHT (1995). O gênero Pheidole, juntamente com Camponotus, é considerado um dos mais amplamente distribuídos (JAFFÉ 1993) e também um dos predominantes em número de espécies no mundo (WILSON 1976). As espécies de Pheidole são ecologicamente diversificadas, incluindo coletoras de sementes, onívoras, predadoras e mutualísticas em associações com plantas e Homoptera (SUDD \& FRANKS 1987; HÖLLDOBLER \& WILSON 1990). Camponotus é considerado um dos gêneros de maior tolerância ecológica (BROWN 1973). Esta característica the confere alto poder de colonização de ambientes.

Embora Wasmannia auropunctata tenha sido relatada como uma espécie capaz de tolerar uma vasta variedade de ambientes (CLARK et. al 1982), os resultados deste trabalho indicam que esta espécie tem uma maior preferência por mata $(30,3 \%$ de freqüência) em relação ao eucaliptal ( $7,0 \%$ de freqüência). O mesmo ocorreu 
com Ectatomma edentatum, que apresentou uma freqüência de 33,8\% na mata, enquanto no eucaliptal sua freqüência foi de apenas $0,4 \%$. Portanto, estas duas espécies são possivelmente mais adaptadas a ambientes heterogêneos de mata que a monoculturas de eucalipto.

As espécies Camponotus rufipes e Solenopsis sp. 2 foram bastante freqüentes nas duas áreas estudadas, embora tenham apresentado uma freqüência de captura pouco maior no eucaliptal. Isto indica que tais espécies sejam bem adaptadas tanto à mata quanto ao eucaliptal, estabelecendo-se de forma bem sucedida nestes dois ambientes.

As formigas da tribo Attini, em especial as espécies dos gêneros Atta e Acromyrmex, são freqüentemente relatadas como pragas de monoculturas agrícolas e florestais. O grande crescimento populacional destas espécies ocorre, principalmente, em monoculturas sem sub-bosques diversificados. Nestas, esses insetos podem tornar-se pragas severas, pois encontram abundante fonte de recurso alimentar e poucos inimigos naturais (ANJos et al. 1993). Diante disto, esperava-se encontrar uma alta freqüência destas formigas no eucaliptal. Contudo, a maior freqüência das Attini foi na área de mata. Na realidade, essas espécies são consideradas habitantes desse ecossistema, somente tornando-se pragas de plantas úteis quando o homem procede aos desmatamentos para formar monoculturas (BENTO \& Della LuCia 1993).

A maior riqueza de espécies obtida para a área de mata, em relação ao eucaliptal, é mais uma evidência de que a formicifauna varia diretamente em função da complexidade estrutural da comunidade vegetal, sendo mais rica, em termos de espécies, em habitats mais heterogêneos (MAJER 1985; BENSON \& HARADA 1988; BURBIDGE et al. 1992; GADAGKAR et al. 1993). Em monoculturas, como o eucaliptal, a diversidade de substratos de nidificação e alimentação é provavelmente menor que a da mata e pode levar a uma redução na riqueza de espécies.

A diferença na riqueza de espécies entre as duas áreas estudadas só foi evidenciada nas armadilhas Doane. Nas armadilhas de iscas, as estimativas da riqueza de espécies das duas áreas não diferiram significativamente entre si. Estes resultados estão possivelmente relacionados com as diferenças de capacidade de amostragem existentes entre as duas técnicas de coletas empregadas. As armadilhas Doane possibilitam uma amostragem mais representativa da riqueza de espécies de formigas que as de iscas. Isto deve-se ao fato de as armadilhas de iscas apresentarem uma seletividade acentuada (CASTRO \& QUEIROZ 1987). Nestas, as formigas interagem diretamente para a defesa do recurso alimentar, só conseguindo permanecer nas placas as espécies capazes de dominar a isca. Portanto, as placas com iscas são mais apropriadas para a amostragem das espécies comportamentalmente dominantes.

Levando-se em consideração as particularidades de cada uma das técnicas de coleta utilizadas, pode-se concluir que não há diferença entre mata e eucaliptal com relação ao número de espécies capazes de dominar as iscas, porém, a comunidade de formigas da mata é possivelmente composta por um maior número de espécies que a do eucaliptal. 
Os dados obtidos neste trabalho podem ser devidos às características idiossincráticas dos locais estudados. Portanto, são necessários estudos onde seja empregado maior número de fragmentos de mata e plantações de eucalipto, para que resultados mais conclusivos e passíveis de generalizações possam ser alcançados.

AGRADECIMENTOS. Os autores agradecem ao Dr. Jacques H. C. Delabie pela grande colaboração na identificação das formigas, ao prof. José Henrique Schoereder pelas críticas e sugestões ao trabalho e ao prof. Paulo De Marco Júnior pelo auxílio na execução das análises estatísticas.

\section{REFERÊNCIAS BIBLIOGRÁFICAS}

ANDERSEN, A.N. 1990. The use of ant communities to evaluate change in Australian terrestrial ecosystems: a review and a recipe. Proc. Ecol. Soc. Aust. 16: 347-357.

ANJos, N.; D.D.O. MoreIRA \& T.M.C. Della LuCIA. 1993. Manejo Integrado de Formigas Cortadeiras em Reflorestamentos, p. 212-241. In: T.M.C. Della LuCIA (Ed.). As Formigas Cortadeiras. Viçosa, Ed. Folha de Viçosa, 262p.

ARMBRECHT, I. 1995. Comparacion de la mirmecofauna en Fragmentos Boscosos del Valle Geográfico del Rio Cauca, Colombia. Bol. Mus. Ent. Univ. Valle. 3 (2): 1-14.

BeLSHAw, R. \& B. Bolton. 1994. A survey of leaf litter ant fauna in Ghana, West Africa (Hymenoptera: Formicidae). Jour. Hym. Res. 3: 5-16.

BENSON, W.W. \& A.Y. HARADA. 1988. Local diversity of tropical and temperate ant faunas (Hymenoptera: Formicidae). Acta Amazon. 18 (3-4): 275-289.

BENTO, J.M.S. \& T.M.C. Della LuCIA. 1993. Acabar com a saúva sem acabar com o Brasil. Ciência Hoje 15 (90): 48-49

Bonnet, A. \& B.C. Lopes. 1993. Formigas de dunas e restingas da Praia da Joaquina, Ilha de Santa Catarina, SC (Insecta: Hymenoptera). Biotemas 6 (1): 107-114.

BROWN JR., W.L. 1973. A comparison of the Hylean and Congo-West African rain forest ant faunas, p.161-185. In: B.J. MEGGERS; E.S. AYENSU \& W.D. DUCKWORTH (Eds). Tropical Forest Ecosystems in Africa and South America: a Comparative Review. Washington, Smithsonian Institution Press, 350p.

BRUYN, L.A.L. 1993. Ant composition and activity in naturally-vegetated and farmland environments on contrasting soils at Kellerbersin, Western Australia. Soil Biol. Biochem. 25 (8): 1043-1056.

Burbidge, A.H.; K. Leicester; S. McDavitT \& J.D. Majer. 1992. Ants as indicators of disturbance at Yanchep National Park, Western Australia. Jour. R. Soc. West. Aust. 75: 89-95.

CASTRO, A.G. \& M.V.B. QueIROZ. 1987. Estrutura e organização de uma comunidade de formigas em agro-ecossistema neotropical. An. Soc. Entomol. Brasil 16 (2): 363-375.

Clark, D.B.; C. Guayasamin; O. Pazmiño; C. Donoso \& Y. PÁez de Villacís. 1982. The tramp ant Wasmannia auropunctata: autoecology and effects on ant diversity and distribution on Santa Cruz Island, Galapagos. Biotropica 14 (13): 


\section{6-207.}

Della Lucia, T.M.C.; M.C. Loureiro; L. Chandler; J.A.H. Freire; J.D. GalVÃo \& B. FERNANDES. 1982. Ordenação de comunidades de Formicidae em quatro agroecossistemas em Viçosa, Minas Gerais. Experientiae 28 (6): 67-94. DOANE, J.F. 1961. Movement on the soil surface, of adult Ctenicera aeripennis destructor (Brow) and Hypolithus bicolor Esch. (Coleoptera: Elateridae), as indicated by funnel pitfall traps, with notes on captures of other arthropods. Can. Entomol. 93: 636-644.

Fowler, H.G.; L.C. Forti; C.R.F. Brandão; J.H.C. Delabie \& H.L. VASCONCELOS. 1991. Ecologia nutricional de formigas, p.131-223. In: A.R. PANNIZI \& J.R.P. PARRA (Eds). Ecologia nutricional de insetos e suas implicações no manejo de pragas. São Paulo, Ed. Manolo, 360p.

Gadagkar, R.; P. NAIR; K. ChandRasheKarA \& D.M. Bhat. 1993. Ant species richness and diversity in some selected localities in Western Ghats, India. Hexapoda 5 (2): 79-94.

Heltshe, J.F. \& N.E. ForRester. 1983. Estimating species richness using the Jackknife procedure. Biometrics 39: 1-11.

Hölldobler, B. \& E.O. Wilson. 1990. The ants. Cambridge, Harvard University Press, 732p.

IBGE. 1959. Enciclopédia dos municípios brasileiros. Rio de Janeiro, Instituto Brasileriro de Geografia e Estatística, vol. 27, 437p.

JAFFÉ, K. 1993. El mundo de las hormigas. Venezuela, Universidad Simón Bolívar, Equinoccio Ediciones, 183p.

LEAL, I.R. \& B.C. LOPES. 1992. Estrutura das comunidades de formigas (Hymenoptera: Formicidae) de solo e vegetação no Morro da Lagoa da Conceição, Ilha de Santa Catarina, SC. Biotemas 5 (1): 107-122.

LOPES, B.C. \& I.R. LEAL. 1991. Levantamento preliminar de formigas (Hymenoptera: Formicidae) de solo e vegetação em um trecho de Mata Atlântica, Morro da Lagoa da Conceição, Ilha de Santa Catarina, SC. Biotemas 4 (2): 51-59.

MAJER, J.D. 1985. Recolonization by ants of rehabilitated mineral sand mines on North Stradbroke Island, Queensland, with particular reference to seed removal. Aust. Jour. Ecol. 10: 31-48.

1992. Ant recolonization of reabilitated bauxite mines of Poços de Caldas, Brasil. Jour. Trop. Ecol. 8: 97-108.

MAJER, J.D. \& A.N. KoCK. 1992. Ant recolonization of sand mines near Richards Bay, South Africa: an evaluation of progress with reabilitation. S. Afr. Jour. Sci. 88 (1): 31-36.

MAJER, J.D. \& P. CAMER-PESCI. 1991. Ants species in tropical australian tree crops and native ecosystems - is there a mosaic? Biotropica 23 (2): 173-181.

MAgurRAN, A.E. 1995. Ecological diversity and its measurement. London, Croom Helm, 179p.

ManLy, B.F.J. 1994. Randomization and Monte Carlo Methods in Biology. New York, Chapmam and Hall, 281 p.

MoRAIS, H.C. \& W.W. BENSON. 1988. Recolonização de vegetação de cerrado após queimada, por formigas arborícolas. Rev. Brasil. Biol. 48 (3): 459-466.

OHMART, C.P.\& P.B. EDWARDS. 1991. Insect herbivory on eucalyptus. Annu. Rev. 
Entomol. 36: 637-657.

Oliveira, M.A. \& T.M.C. Della LuCia. 1992. Levantamento de Formicidae de chão em áreas mineradas sob recuperação florestal de Porto Trombetas, Pará. Bol. Mus. Para. Emílio Goeldi, sér. Zool., 8 (2): 375-384.

SUDD, J.H. \& N.R. FRANKS. 1987. The behavioural ecology of ants. New York, Chapman and Hall, 206p.

WiLson, E.O. 1971. The insect societies. Cambridge, Massachusetts, Belknap Press of Harvard University Press, 548p.

1976. Which are the most prevalent ant genera? Stud. Entomol. 19 (1-4): $187-200$.

Recebido em 02.V.1997; aceito em 03.XI.1998. 